gene encoding the human form of GTP cyclohydrolase-was significantly associated with low scores for leg pain following diskectomy for persistent radicular low back pain $(P=0.009)$. Healthy individuals homozygous for this haplotype exhibited a reduced response to experimental pain, and a smaller upregulation of $\mathrm{GCH} 1$ in response to a forskolin challenge, compared with controls. The researchers conclude that $\mathrm{BH} 4$ is a regulator of pain sensitivity and suggest that a strategy to reduce excess de novo synthesis of $\mathrm{BH} 4$ might be useful in the management or prevention of chronic pain.

Original article Tegeder I et al. (2006) GTP cyclohydrolase and tetrahydrobiopterin regulate pain sensitivity and persistence. Nat Med 12: 1269-1277

\section{No evidence of a neuroprotective effect for TCH346 in Parkinson's disease}

Currently, there is no available treatment for the prevention or modification of Parkinson's disease (PD) progression. Therapy typically consists of a dopamine replacement strategy, which is often associated with long-term complications such as motor dyskinesias. Development of a neuroprotective treatment for PD would thus be highly desirable. Disappointing results were recently reported, however, for a large prospective double-blind trial of TCH346, a drug found to be strongly neuroprotective in laboratory animals.

Olanow et al. recruited 301 patients with early untreated PD, 255 of whom completed the study. Participants were randomized to receive either placebo, or $\mathrm{TCH} 346$ at a daily dose of $0.5 \mathrm{mg}, 2.5 \mathrm{mg}$ or $10 \mathrm{mg}$, for 12-18 months. Patients were assessed at regular intervals using the Unified Parkinson's Disease Rating Scale (UPDRS) and the Parkinson's Disease Questionnaire (PDQ)-39 scale-a measure of disease-specific quality of life. The primary end point was the time to development of a disability that required dopaminergic treatment.

There were no significant differences between study groups regarding the primary outcome. There were also no significant differences between groups with regard to the annual rate of change in UPDRS and PDQ-39 scores, with the exception of some subcomparisons.

The authors speculate that the discrepancy between the laboratory and clinical findings could relate to a number of factors, such as the intrinsic difficulties in predicting brain bioavailability for a given dose of TCH346, and the lack of clinical markers to indicate the appropriate dose in humans. They suggest that animal models more directly reflecting PD pathogenesis should be developed in future.

Original article Olanow CW et al. (2006) TCH346 as a neuroprotective drug in Parkinson's disease: a double-blind, randomized, controlled trial. Lancet Neurol 5: 1013-1020

\section{Patients with imploding migraine headache respond to botulinum A toxin therapy}

Analyses of pooled data have failed to demonstrate a superiority of local intramuscular injections of botulinum toxin type A (BTX-A) over placebo for the prophylaxis of migraine; however, many clinicians have noted marked reductions in the frequency of migraine attack in certain patients following BTX-A therapy. Jakubowski et al. carried out a prospective study to identify neurological markers that might distinguish between responders and nonresponders.

Each participant $(n=42)$ received 100 units of BTX-A administered as 21 extracranial injections. Between weeks 4 and 12 following BTX-A treatment, 27 of the cohort could clearly be classified as responders $(n=14)$ or nonresponders $(n=13)$. Those responsive to BTX-A treatment reported reductions in attack frequency, attack duration, and headache intensity. The incidences of neck muscle tenderness, cutaneous allodynia and migraine symptoms were similar in responders and nonresponders. Notably, however, responders and nonresponders could be split into two groups on the basis of the description of their pain-93\% of individuals responsive to BTX-A therapy described their migraine pain as either imploding (crushing) or ocular (eye-popping) headache, whereas 100\% of nonresponders described a build-up of pressure inside their head (exploding headache). This result was retrospectively validated in an independent cohort of 36 migraine patients who had received BTX-A therapy. The amelioration of imploding headaches by BTX-A therapy indicates that this type of migraine pain is mediated, at least in part, by extracranial innervation.

Original article Jakubowski M et al. (2006) Exploding vs. imploding headache in migraine prophylaxis with Botulinum Toxin A. Pain 125: 286-295 